\title{
Synthesis and Properties of Organic-Inorganic Hybrid Porous Polymers Obtained with Click Addition Reactions of Thiol-Functionalized Random Type Silsesquioxane by and Diacrylate or Diisocyanate Compounds
}

\author{
Tomoharu Miyanaga ${ }^{1}$, Naofumi Naga ${ }^{1,2 *}$ \\ ${ }^{1}$ Graduate School of Engineering and Science, Shibaura Institute of Technology, Tokyo, Japan \\ ${ }^{2}$ Department of Applied Chemistry, College of Engineering, Shibaura Institute of Technology, \\ Tokyo, Japan \\ Email: ^nnaga@sic.shibaura-it.ac.jp
}

How to cite this paper: Miyanaga, T. and Naga, N. (2020) Synthesis and Properties of Organic-Inorganic Hybrid Porous Polymers Obtained with Click Addition Reactions of Thiol-Functionalized Random Type Silsesquioxane by and Diacrylate or Diisocyanate Compounds. Open Journal of Polymer Chemistry, 10, 1-20.

https://doi.org/10.4236/ojpchem.2020.101001

Received: February 1, 2020

Accepted: February 16, 2020

Published: February 19, 2020

Copyright $\odot 2020$ by author(s) and Scientific Research Publishing Inc. This work is licensed under the Creative Commons Attribution International License (CC BY 4.0).

http://creativecommons.org/licenses/by/4.0/

\begin{abstract}
Organic-inorganic hybrid network polymers have been synthesized by addition reaction of a thiol-functionalized random type silsesquioxane (SQ109) and alkyl diacrylate or diisocyanate compounds. Thiol-ene reaction of SQ109 and 1,4-butanediol diacrylate (BDA) successfully yield porous polymer in toluene initiated by azobis(isobutyronitrile) (AIBN) at $60^{\circ} \mathrm{C}$. Morphology of the porous polymers was composed by connected globules, and the diameter of the globules decreased with increasing in the monomer concentration of the reaction system. By contrast, the reaction with 1,6-hexanediol diacrylate or 1,5-hexadiene yielded homogeneous clear gels. Thermal analyses of SQ109-BDA porous polymers indicated that thermal degradation of ester groups of BDA in the polymer network occurred at around $300^{\circ} \mathrm{C}$. The porous polymer was also obtained by the reaction using a photo-initiator (Irugacure184) at room temperature, and showed higher Young's modulus than the corresponding porous polymer obtained with the reaction with AIBN due to the small size of the globules. Young's modulus of SQ109-BDA porous polymer increased with increasing in the monomer concentration of the reaction systems. Thiolisocyanate addition reactions between SQ109 and hexamethylene diisocyanate (HDI) or methylenediphenyl 4,4'-diisocyanate (MDI) were investigated to obtain network polymers. The reactions in toluene yielded the corresponding homogeneous clear gels. By contrast the reactions in a mixed solvent of toluene (50 vol.\%) and $N, N$-dimethylformamide (50 vol.\%) produced porous po-
\end{abstract}


lymers. The morphology of the porous polymers was composed by connected globules or aggregated particles. The size of globules and particles in the SQ109-HDI porous polymers was larger than those in the SQ109-MDI porous polymers. Thermal degradation of SQ109-HDI and SQ109-MDI porous polymers started at round $260^{\circ} \mathrm{C}$ and showed endothermic peak at around $350^{\circ} \mathrm{C}$ derived from degradation of thio-urethane bond.

\section{Keywords}

Silsesquioxane, Thiol, Diacrylate, Diisocyanate, Gel, Porous Polymer, Morphology

\section{Introduction}

Silsesquioxane is general term for materials, which are formed by Si-O back bone with $\left(\mathrm{RSiO}_{1.5}\right)_{\mathrm{n}}$ composition. Silsesquioxane is one of the most useful building blocks to obtain organic-inorganic hybrid polymers. There are some types of silsesquioxanes, such as cage (perfect, imperfect), random, and ladder, as shown in Scheme 1. The cage type silsesquioxanes have been widely used for synthesis of the hybrid polymers with network structure. We reported synthesis of gels by a hydrosliylation reaction of a perfect cage type silsesquioxanes, as a joint monomer, and $\alpha, \omega$-diolefin, as a linker monomer, using Pt catalysts in toluene, [1] [2] [3]. The type of gel was also synthesized from octavinyloctasilsesquioxane, with $\alpha$, $\omega$-alkyldithiol or $\alpha$, $\omega$-alkyldiazide using non catalytic thiol-ene reaction or azide-alkene reaction [4]. Addition reactions with multifunctional silsesquioxane and $\alpha, \omega$-bifunctional monomer, so called joint and liker concept, makes it possible to form network in the organic-inorganic hybrid gels with homogeneous structure, controlled mesh size, and designed molecular geometry.

We have been developing various types of network polymers based on the joint and linker concept, and recently found some reaction systems, which yielded porous polymers. For example, Michael addition reaction of multifunctional acrylate and $\alpha, \omega$-dithiol or diamine in dimethyl sulfoxide, or thiol-isocyanate reaction of multifunctional thiol and $\alpha, \omega$-diisocyanate in toluene yielded porous polymers via polymerization induced phase separation [5] [6]. Morphology of the porous polymers, co-continuous monolithic structure, or globules, could be controlled by the reaction conditions, such as monomer concentration, reaction temperature etc. Porous polymers have been applied in column of separation, support of catalysts, scaffold for cell cultivation and so on (for example [7] [8]). Organic-inorganic hybrid porous polymers should be attractive materials due to their characters derived from both the organic and inorganic moieties. An organic-inorganic hybrid porous polymer was prepared by thiol-ene reaction of a perfect cage type octavinyloctasilsesquioxane and multi-functional thiol in the presence of polyethylene glycol (PEG) as pore generator (porogen) [9]. There 

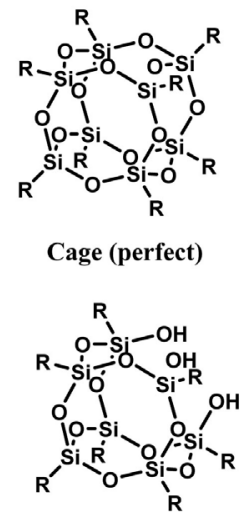

Cage (imperfect)
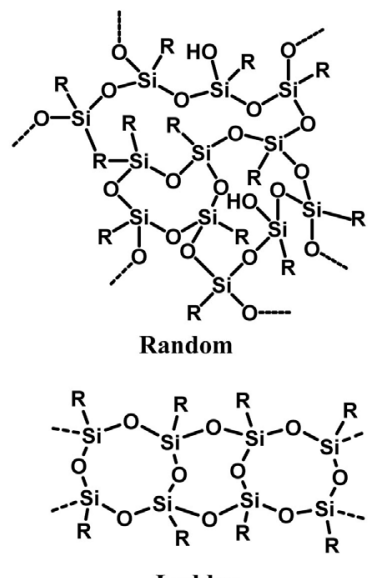

Ladder

Scheme 1. Structure of silsesuquioxanes.

would be two issues to make the porous polymer fit for practical use. One is removal of PEG as the porogen from the resulting porous polymers. Contamination of porogen in the polymers should lower performance of the porous polymer. Another is hard availability of perfect cage type silsesquioxane. Although perfect cage type silsesquioxanes are available as research reagents from some chemical companies, the prices are usually high for commercial use. Perfect cage type silsesquioxane must be suitable to obtain the porous polymer due to the rigidity of its chemical structure. By contrast, an imperfect cage type silsesquioxane was used to prepare a soluble network polymer [10]. We focused on commercial availability of silsesquioxane, and selected random type silsesquioxane, as shown in Scheme 1. The random type silsesquioxane is produced industrially, and widely used as a monomer of coatings. Random type silsesquioxane has flexible chemical structure and may not be suitable to obtain rigid porous polymer. We came to an idea that phase control, homogeneous or phase separation, during network formation with random type silsesquioxane should make it possible to obtain porous polymer even from the random type silsesquioxane due to its multi-functionality.

In this paper, to begin with, we report synthesis of organic-inorganic hybrid network polymers by thiol-ene reaction of random type silsesquioxane having thiol group and diacrylate using radical initiators (Scheme 2, Scheme 3(A)). The thiol group also shows high reactivity with isocyanate, which are useful to obtain network polymers [11] [12] [13] [14]. The organic-inorganic hybrid network polymers have been also synthesized from diisocyanate compounds as the linker monomers, as shown in Scheme 2 and Scheme 3(B). We study effect of molecular structure of the $\alpha$, $\omega$-bifunctional monomer, features of the solvents, and monomer concentration of the reaction system on phase of the reaction system and morphology of porous polymers. Morphology of all the porous polymers should be induced by phase separation via spinodal decomposition. Their phase and porous morphology (structure and size) were discussed based on affinity between the networks and solvents, and correlation of polymerization rate 

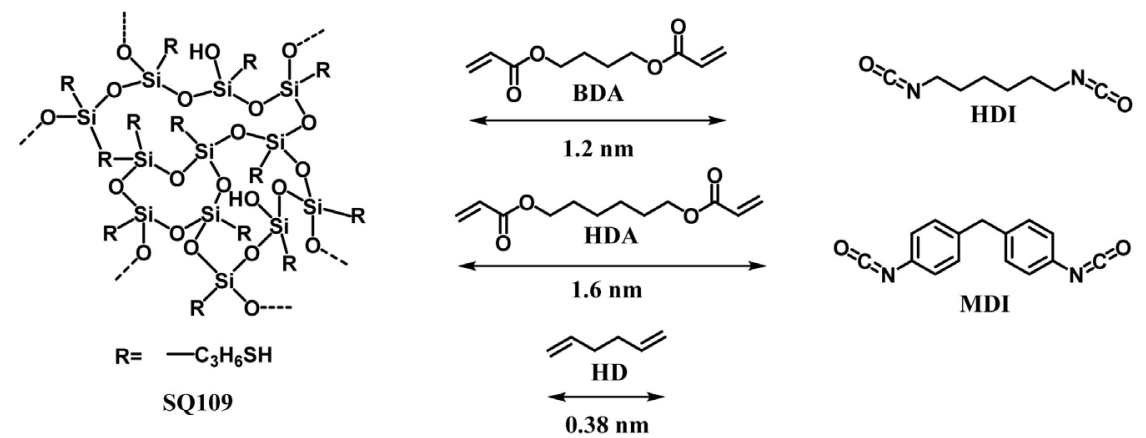

Scheme 2. Joint (random type thil functionalized silsesquioxane, SQ109) and linker monomers used in the present research.
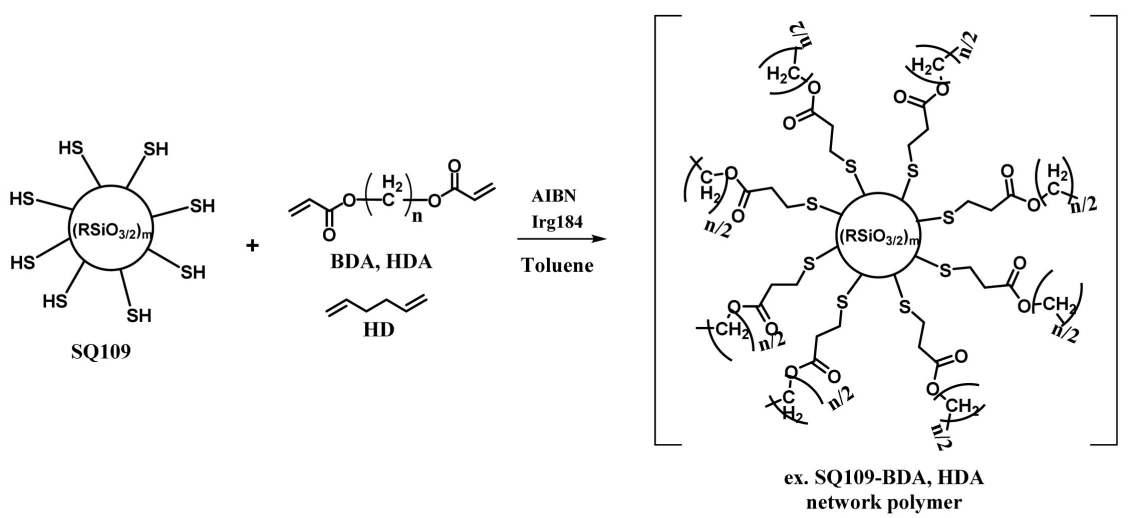

(A)
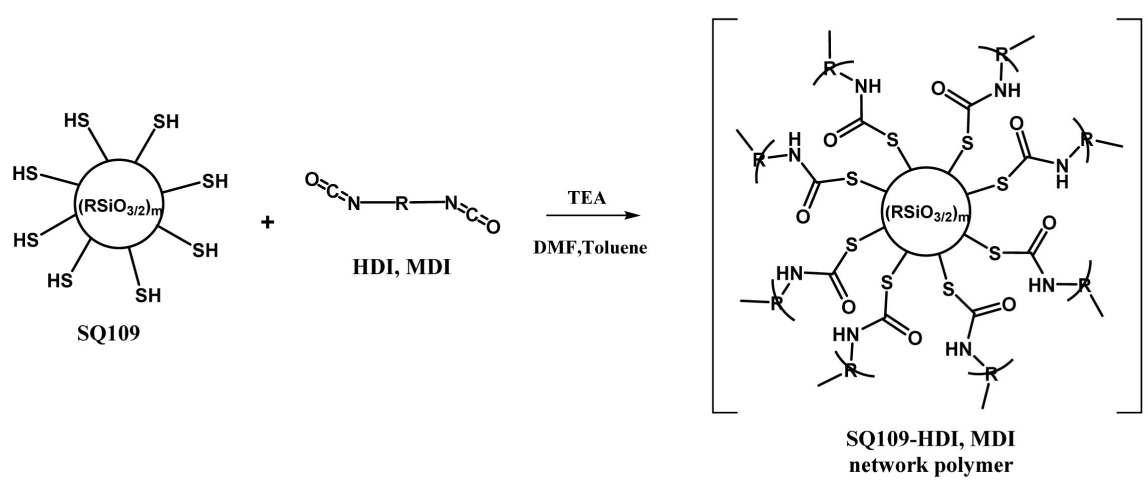

(B)

Scheme 3. Model of joint-linker type network polymers synthesized by (A) thiol-ene reaction of random type silsesquioxane (SQ109) and diacrylate (BDA, HDA) or diolefine (HD); (B) thiol-isocyanate (HDI, MDI) reaction of SQ109 and diisocyanate.

and phase separation rate in the reaction systems. Thermal and mechanical properties of the porous polymers were also studied based on their chemical and porous structures.

\section{Method}

\subsection{Materials}

Thiol containing random-type silsesquioxane (Conposeran SQ109, SH: 152.4 g/eq., 
propylene glycol methyl ether acetate (PGMEA) solution) was kindly donated from Arakawa Chemical Co. Ltd., and used after evaporation of PGMEA and drying in vacuo. at $80^{\circ} \mathrm{C}$ for 48 h. 1,4-Butanediol diacrylate (BDA) and 1,6hexanediol diacrylate (HDA) were commercially obtained from Aldrich Chemical Co. Ltd., and were purified by passing through $\mathrm{Al}_{2} \mathrm{O}_{3}$ column before use to remove polymerization inhibitors. 1,5-Hexadiene (HD, Tokyo Chemical Industry Co., Ltd.) was dried with $\mathrm{CaH}_{2}$ and distilled under nitrogen atmosphere before use. Hexamethylene diisocyanate (HDI, Tokyo Chemical Industry Co., Ltd.) and methylenediphenyl 4,4'-diisocyanate (MDI, Kanto Chemical Co., Inc.) were commercially obtained, and used as received. Azobis(isobutyronitrile) (AIBN, Kanto Chemical Co., Inc.), Irugacure184 (Irg184, Chiba Specialty Chemicals), and triethylamine (TEA, Kanto Chemical Co., Inc.) were commercially obtained, and used without further purification. High purity grade of toluene, $N, N$-dimethylformamide (DMF) and acetone were commercially obtained from Kanto Chemical Co., Inc., and used as received.

\subsection{Synthesis of Network Polymers}

The molar ratio of S-H group in SQ109 to acrylate, vinyl, or isocyanate group in the linker molecule was adjusted to 1.0 .

\subsubsection{Synthesis of Network Polymers by Means of Thiol-Ene Reaction of SQ109 and BDA, HDA, or HD}

Thiol-ene reaction of SQ109 with BDA (Run 2) is described as an example. SQ109 (0.36 g, SH $2.4 \mathrm{mmol})$, BDA (0.24 g, $1.2 \mathrm{mmol})$, toluene $(2.5 \mathrm{~mL})$, and AIBN (7.4 mg, $0.045 \mathrm{mmol}$ ) were added to a glass ample tube (diameter $13 \mathrm{~mm}$, $10 \mathrm{~mL}$ ). The reaction system was stirred by vortex mixer for several minutes. After the sample tube was sealed by burning off, the reaction system was kept at $60^{\circ} \mathrm{C}$ for $24 \mathrm{~h}$. The reaction with HDA or HD was conducted under the same procedures.

SQ109 (0.36 g, SH $2.4 \mathrm{mmol})$, BDA (0.24 g, $1.2 \mathrm{mmol})$, toluene $(2.5 \mathrm{~mL})$, and Irg184 (9.2 $\mathrm{mg}, 0.045 \mathrm{mmol}$ ) were added to a glass ample tube (diameter $13 \mathrm{~mm}$, $10 \mathrm{~mL}$ ). The reaction system was stirred by vortex mixer for several minutes. After the sample tube was sealed by burning off, UV of $365 \mathrm{~nm}, 30 \mu \mathrm{W} / \mathrm{cm}^{2}$ was irradiated for $20 \mathrm{~min}$ at room temperature. The reaction system was kept standing for $24 \mathrm{~h}$ without $\mathrm{UV}$ irradiation at room temperature.

\subsubsection{Synthesis of Network Polymers by Means of Addition Reaction of SQ109 and HDI or MDI}

Addition reaction of SQ109 with HDI (Run 16) in DMF/toluene is described as an example. SQ109 (0.48 g, SH $3.2 \mathrm{mmol})$, HDI (0.27 g, $1.6 \mathrm{mmol})$, and DMF/ toluene (50/50 vol.\%/vol.\%) mixed solvent (2.4 g) were added to a glass ample tube (diameter $13 \mathrm{~mm}, 10 \mathrm{~mL}$ ). The reaction system was stirred by vortex mixer for several minutes. After addition of TEA $(20.0 \mu \mathrm{L}, 0.14 \mu \mathrm{mol})$ to the reaction solution, the sample tube was sealed by burning off, the reaction system was kept 
at room temperature for $24 \mathrm{~h}$. The reaction with MDI or in toluene was conducted under the same procedures.

The obtained porous polymer was soaked in methanol with ultrasonification for $6 \mathrm{~h}$ to replace the solvent, and dried at room temperature under normal pressure following in vacuo for $4 \mathrm{~h}$.

\subsection{Analytical Procedures}

FT-IR spectra of the network polymers were recorded on a Shimadzu IR Affinity-1S using attenuated total reflectance (ATR) unit, and 20 scans were accumulated from 4000 to $400 \mathrm{~cm}^{-1}$.

Scanning electron microscopy (SEM) images of the porous polymers were obtained by a JEOL JSM-7610F microscope with a LEI detector at an acceleration voltage of $3.0 \mathrm{kV}$. Solubility parameter (SP) value of toluene and DMF mixed solvent was calculated using a following equation,

$$
\sigma_{\text {mix }}=\sigma_{1} \cdot \varphi_{1}+\sigma_{2} \cdot \varphi_{2}
$$

where $\sigma$ or $\varphi$ is SP value (toluene: $8.8\left(\mathrm{cal} / \mathrm{cm}^{3}\right)^{1 / 2}$, DMF: $\left.12.0\left(\mathrm{cal} / \mathrm{cm}^{3}\right)^{1 / 2}\right)$ or volume ratio (50 vol.\%/50 vol.\%) of solvent.

Thermogravimetric analysis of the polymers was conducted with a Bruker AXS TG-DTA2020SA. The sample was heated from room temperature to $480^{\circ} \mathrm{C}$ at a rate of $10^{\circ} \mathrm{C} / \mathrm{min}$ under argon atmosphere. Thermal analysis of the polymers was conducted with a differential scanning calorimetry (DSC) of a Rigaku DSC 8230. The sample was heated from room temperature to $500^{\circ} \mathrm{C}$ at a rate of $10^{\circ} \mathrm{C} /$ min under nitrogen atmosphere.

Mechanical properties of the porous polymers were investigated by the compression test with Tensilon RTE-1210 (ORIENTEC Co. LTD.). The test samples were cut to $0.3-0.5 \mathrm{~cm}$ cube, and pressed at a rate of $0.5 \mathrm{~mm} / \mathrm{min}$ at room temperature.

\section{Results and Discussion}

\subsection{SQ109-Alkyl Diacrylate, Diolefin Network Polymer}

Thiol-ene reaction of SQ109 and BDA was conducted in toluene initiated by AIBN at $60^{\circ} \mathrm{C}$ under various monomer concentrations, Scheme $3(\mathrm{~A})$, and the results are summarized in Table 1 . The reaction systems containing $5 \mathrm{wt} \%$ of the monomers yielded white precipitate. White gels were obtained in the reactions containing 15, 25, and $35 \mathrm{wt} \%$ monomers. By contrast, clear gel was yielded in the reaction system containing $45 \mathrm{wt} \%$ monomers. Figure 1 shows photos of dried SQ109-BDA network polymers obtained from the reaction systems with 15 $w t \%$ (run 2) and $45 \mathrm{wt} \%$ (run 5) monomers after drying. The appearance of former or latter polymer is white or clear, respectively. Figures 2(A)-(D) show SEM images of the dried SQ109-BDA network polymers obtained from the reaction systems with different monomer concentrations. The network polymer obtained from the reaction system containing $5 \mathrm{wt} \%$ monomers showed a morphology, 


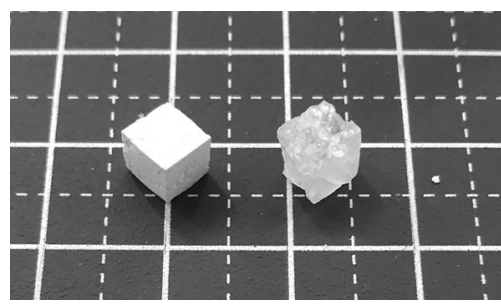

Figure 1. Photos of QS109-BDA network polymers, monomer concentration in reaction solution; $15 \mathrm{wt} \%$ (rub 2, right), $45 \mathrm{wt} \%$ (run 5, left), bold scale: $1 \mathrm{~cm}$.

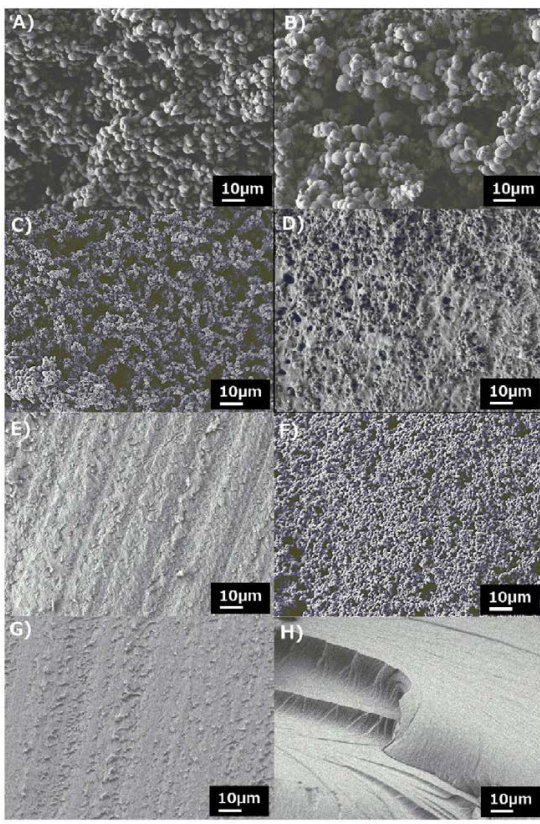

Figure 2. SEM images of SQ109-BDA, SQ109-HDA, and SQ109-HD polymers synthesized by thiol-ene reaction with AIBN at $60^{\circ} \mathrm{C}$, Solvent: toluene; SQ109-BDA monomer concentration (A) $5 \mathrm{wt} \%$, (B) $15 \mathrm{wt} \%$, (C) $25 \mathrm{wt} \%$, (D) $35 \mathrm{wt} \%$, (E) $45 \mathrm{wt} \%$, (F) SQ109-BDA 15 wt\% with $\operatorname{Irg} 184$ under UV reaction at room temp., (G) SQ109-HDA 25 wt\%, (H) SQ109-HD 25 wt\%.

Table 1. Synthesis of network polymers by thiol-ene reaction of SQ109 with BDA, HDA, or HD in toluene.

\begin{tabular}{ccccccc}
\hline Run & Linker & Initiator & $\begin{array}{c}\text { Monomer } \\
\text { conc. }(\text { wt } \%)\end{array}$ & State & $\begin{array}{c}\text { SEM } \\
\text { observation }\end{array}$ & $\begin{array}{c}\text { Size }^{\mathrm{a}} \\
(\mu \mathrm{m})\end{array}$ \\
\hline 1 & BDA & AIBN & 5 & White precipitate & Porous & 3.6 \\
2 & BDA & AIBN & 15 & White gel & Porous & $1.7,5.0$ \\
3 & BDA & AIBN & 25 & White gel & Porous & 1.1 \\
4 & BDA & AIBN & 35 & White gel & Porous & 1.5 \\
5 & BDA & AIBN & 45 & Clear gel & Bulk & - \\
$6^{\text {b }}$ & BDA & Irg184 & 15 & White gel & Porous & 1.4 \\
7 & HDA & AIBN & 25 & Clear gel & Bulk & - \\
8 & HD & AIBN & 25 & Clear gel & Bulk & - \\
\hline
\end{tabular}

a averaged diameter of globule, ${ }^{b}$ reaction conditions, room temp, UV irradiation time $20 \mathrm{~min}$. 
which was formed by connected (almost) sphere globules with averaged radius of $3.6 \mu \mathrm{m}$ deterimned by image processing. The connected globules formed co-continuous vacant space in the porous structure. The reaction system with 15 $\mathrm{wt} \%$ monomers yielded the porous polymer formed by connected sphere globules with small $(1.7 \mu \mathrm{m})$ and large $(5.0 \mu \mathrm{m})$ diameters. The network polymer obtained from the reaction system with $25 \mathrm{wt} \%$ formed porous structure composed by distorted small globules about $1.1 \mu \mathrm{m}$. These structures should be formed by polymerization induced phase separation via spinodal decomposition. The averaged globule diameter tended to decrease with increasing in the monomer concentration of the reaction system as observed in the SEM images of the polymers obtained from reaction systems with $5 \mathrm{wt} \%$ and $25 \mathrm{wt} \%$ monomers, and summarized in Table 1 . The polymer obtained from the reaction system with $35 \mathrm{wt} \%$ monomers showed coexistence of co-continuous (hole) and connected globules. A model of formation of porous polymer to explain the results is shown in Figure 3. In the early stage of the phase separation via spinodal decomposition, co-continuous monolithic structure is formed, Figure 3(ii). The co-continuous structure transfers to droplets, which forms small particles or globules, by interfacial tension as show in Figure 3(iii). The size of the droplets increases as the reaction goes on, Figure 3(iv). The reaction system with $5 \mathrm{wt} \%$ monomers would form the morphology of the connected globules with relatively large averaged diameter of $3.6 \mu \mathrm{m}$ at the state of Figure 3(iv). Increase of the monomer concentration in the reaction system should increase the reaction rate and fixed the porous structure at earlier state of phase separation, as shown in Figure 3(iii). Actually, increase of the monomer concentration in the reaction system tended to decrease the diameter of globules (averaged diameter: $1.1 \mu \mathrm{m}$ ), as observed in the porous polymer obtained from the reaction system with 25 $\mathrm{wt} \%$ monomers. Size distribution of diameter of the globules, co-existence of small $(1.7 \mu \mathrm{m})$ and large $(5.0 \mu \mathrm{m})$ globules, were observed in the porous polymer obtained from the reaction system with $15 \mathrm{wt} \%$ monomers. One explanation for the result may be inhomogeneity of the phase separation in the reaction. The small globules would be formed at the early stage of the polymerization under high monomer concentration. Monomer concentration in the reaction decreased with increasing in polymerization time, and large globules would be yielded at the late stage of the polymerization. Coexistence of different monomer concentrations in the reaction system derived from phase equilibrium might be another account. Coexistence of co-continuous (hole) and globule structures in the porous polymer obtained from the reaction system with 35 wt $\%$ monomers would be induced at the phase separation state between Figure 3(ii) and Figure 3(iii). Further increase of the monomer concentration in the reaction system ( $45 \mathrm{wt} \%$ ) should increase the reaction rate and formed the network polymer before phase separation as clear gel, Figure 3(i), and showed non-porous morphology. 


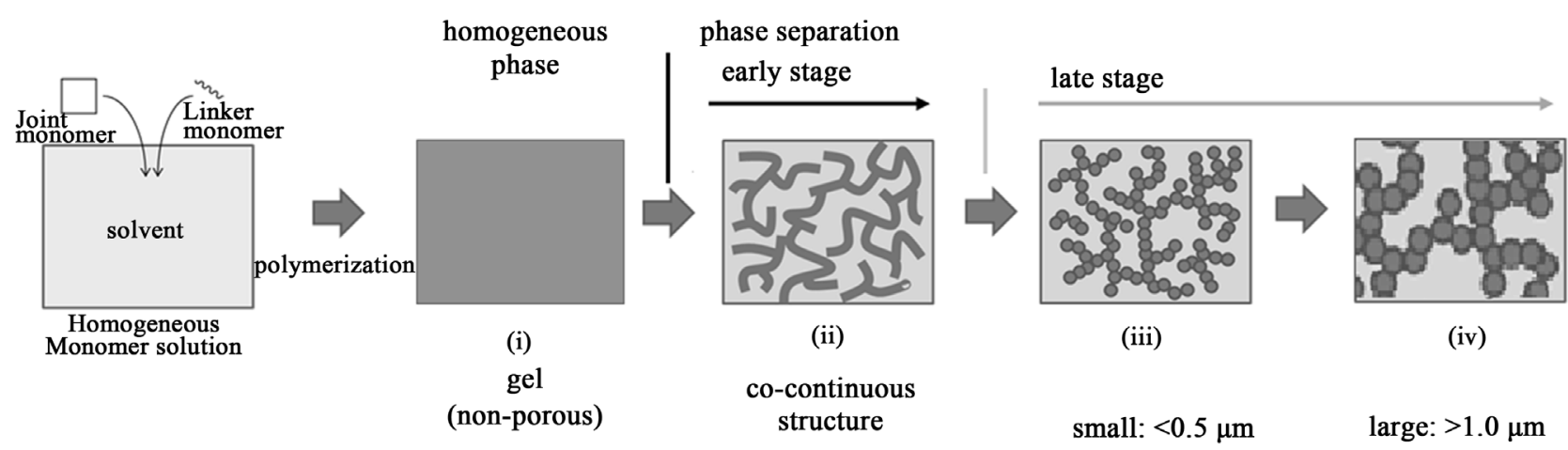

Figure 3. A model of network formation process in the reaction of joint (SQ109) and linker (bi-functional) monomers.

Thiol-ene reaction of SQ109 and BDA (monomer concentration: $15 \mathrm{wt} \%$ ) initiated by Irg184 with UV irradiation at room temperature also yielded the porous polymer. The diameter of the globules in the porous polymer $(1.4 \mu \mathrm{m})$ was smaller than that of the corresponding polymer prepared by the reaction initiated by AIBN at $60^{\circ} \mathrm{C}$. Lower reaction temperature of the reaction with $\operatorname{Irg} 184$ should decrease solubility of the intermediated polymer network and formed the porous structure at earlier stage.

Thiol-ene reaction of SQ109-HDA or SQ109-HD formed clear gel and yielded bulk polymer with non-porous morphology, as shown in Figure 2(G) or Figure 2(H), respectively. Molecular structure of SQ109-BDA, SQ109-HDA, and SQ109HD polymer was studied by FT-IR spectroscopy, as shown in Figure 4. The absorption peaks at around $1620 \mathrm{~cm}^{-1}$ derived from alkene (acrylate or vinyl group in BDA, HDA, or HD) and $2560 \mathrm{~cm}^{-1}$ derived from thiol group (in SQ109) were almost disappeared in the spectra of the resulting network polymers. Absorption peaks derived from Si-O-Si unit of SQ109 were detected at around $1050-1200 \mathrm{~cm}^{-1}$. SQ109-BDA and SQ109-HDA polymers showed absorption peaks derived from carbonyl group at around $1700 \mathrm{~cm}^{-1}$ (Spectra data were obtained from Wiley Subscription Services, Inc.). These results showed the thiol-ene reaction successfully occurred and formed the corresponding polymer networks in all the reaction systems. In other words, difference in morphology of the network polymers should not be derived from difference in the reaction conversion. The difference in the morphology can be explained by affinity between the network and toluene in the reaction system. SP values of connection moiety in SQ109-BDA, SQ109-HDA, and SQ109-HD network polymers can be calculated by Fedors' method, and are shown in Scheme 4 [15]. The calculated SP value of connection moiety, boxed part in Scheme 4 , formed by the addition of thiol with BDA (acrylate) or $\mathrm{HD}$ (vinyl) is 10.5 or $8.6\left(\mathrm{cal} / \mathrm{cm}^{3}\right)^{1 / 2}$, respectively. The SP of toluene is $8.9\left(\mathrm{cal} / \mathrm{cm}^{3}\right)^{1 / 2}$. Small difference in SP values between the thio-alkyl unit and toluene should induce homogeneous phase, clear gel, in the polymerization of SQ109 and HD in toluene. The calculated SP of connection moiety in SQ109-HDA network polymer, $10.2\left(\mathrm{cal} / \mathrm{cm}^{3}\right)^{1 / 2}$, is close to that of SQ109-BDA network polymer. One explanation for the difference in morphology 


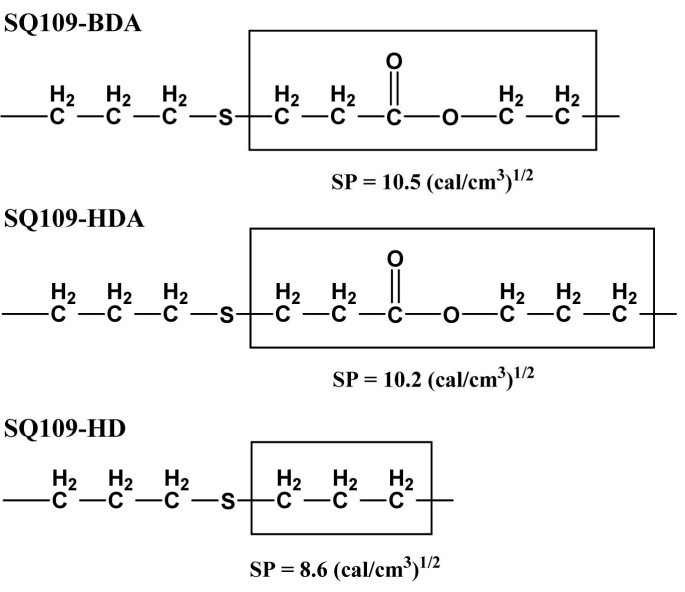

Scheme 4. Structure and SP value of connection moiety in SQ109-BDA, SQ109-HDA, and SQ109-HD network polymers.

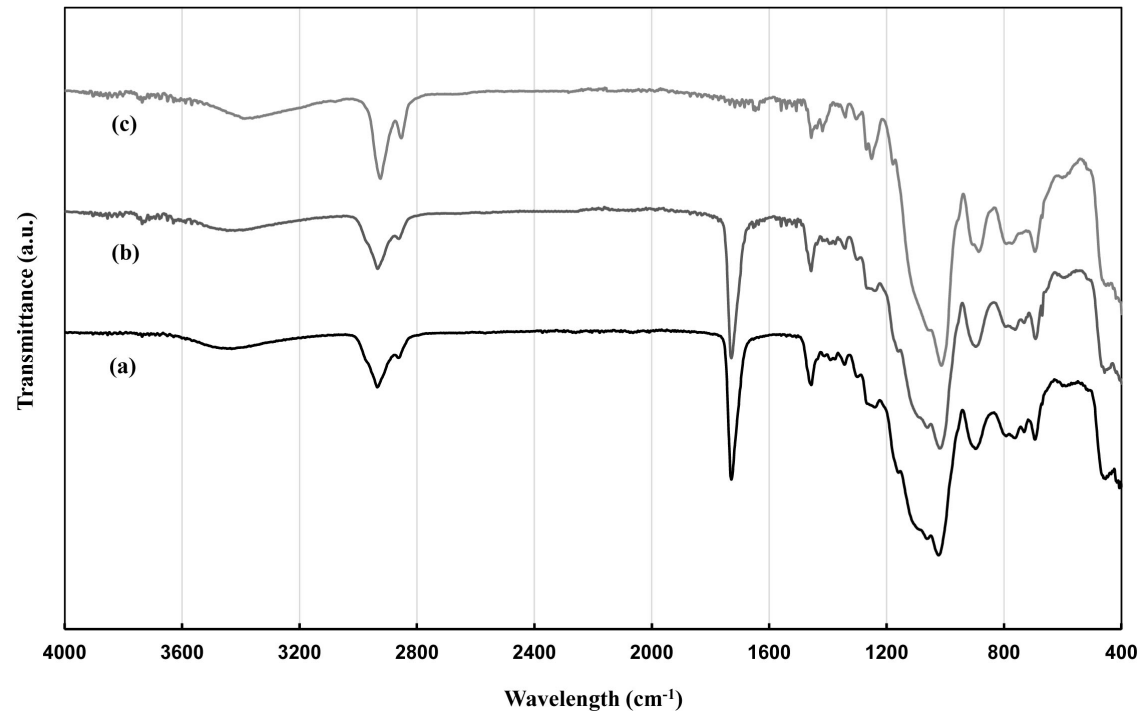

Figure 4. FT-IR spectra of (a) SQ109-BDA; (b) SQ109-HDA; and (c) SQ109-HD network polymers, monomer concentration in the reaction system; $25 \mathrm{wt} \%$.

between SQ109-BDA and SQ109-HDA network polymers may be that larger molecular size of HDA (calculated extended molecular length: $1.6 \mathrm{~nm}$ ) than BDA (1.2 nm), as shown in Scheme 2, would promote permeation of toluene into SQ109-HDA network, which should induce swelling of network as homogeneous clear gel.

Thermal properties of SQ109-BDA network polymers were investigated by TGA and DSC analyses, as shown in Figure 5. Weight loss of SQ109-BDA network polymer (run 2) began at round $300^{\circ} \mathrm{C}(5 \mathrm{wt} \%$ weight loss was attained at $314^{\circ} \mathrm{C}$ ) in TGA analysis. Endothermic peaks were detected at around $360^{\circ} \mathrm{C}$ in DSC profiles independent of the monomer concentration in the reaction system and kinds of the initiator used. These phenomena should be derived from thermal degradation of ester group of BDA in the polymer network. Broad shoulder endothermic peaks were detected at around $420^{\circ} \mathrm{C}$ in all the DSC profiles of 


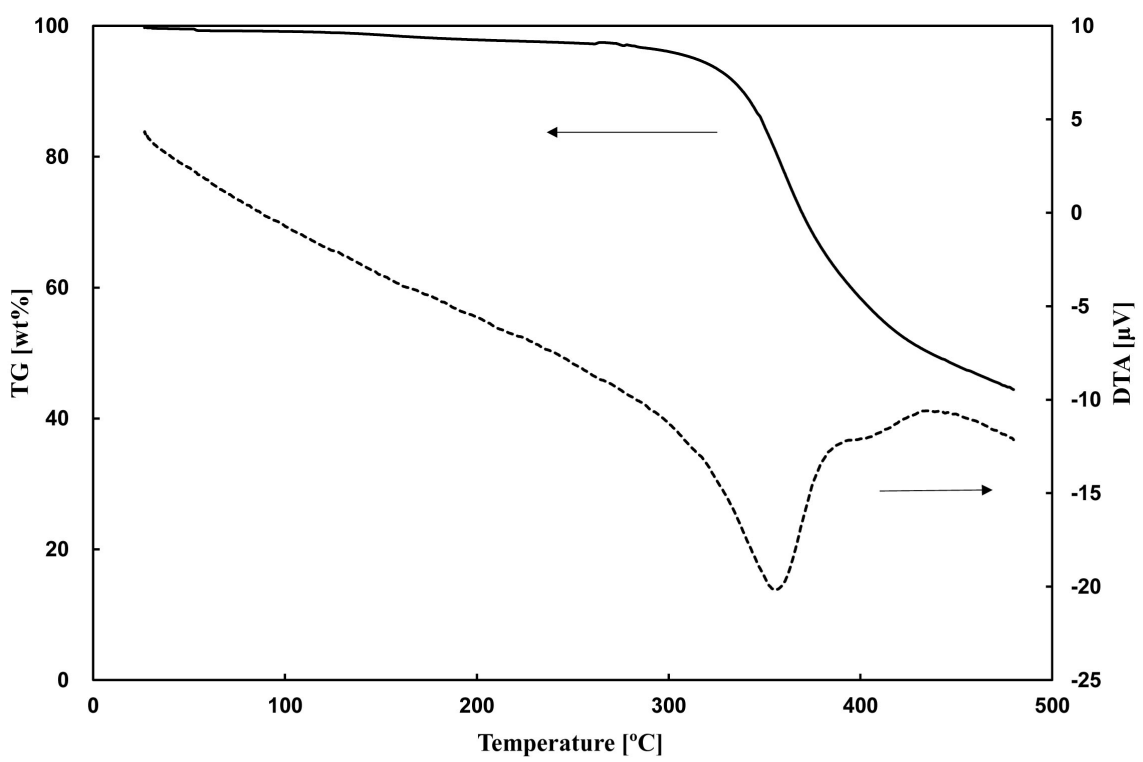

(A)

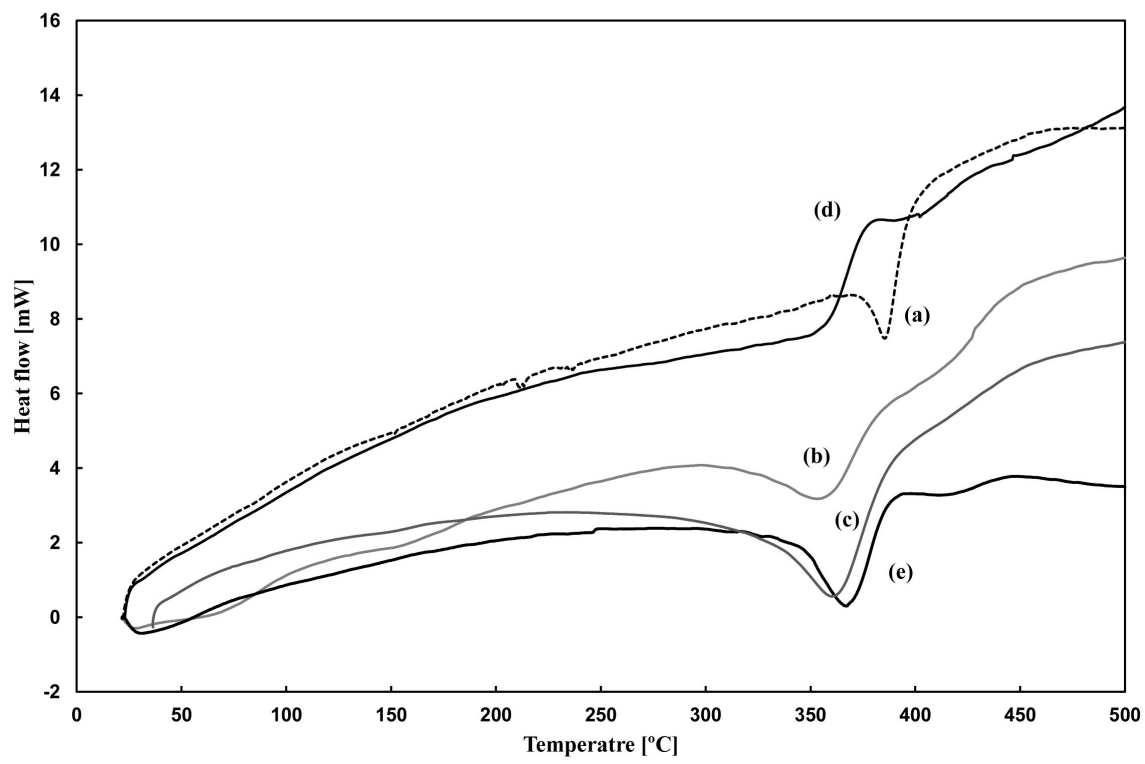

(B)

Figure 5. (A) TG-GTA profile (run 2) and (B) DSC profiles of (a) SQ109 for a reference and SQ109-BDA network polymers; monomer concentration in the reaction system, (b) $15 \mathrm{wt} \%$ (run 2), (c) $25 \mathrm{wt} \%$ (run 3), or (d) $45 \mathrm{wt} \%$ (run 5) initiated by AIBN, and (e) 15 wt\% initiated by $\operatorname{Irg} 184$ (run 6).

SQ109-BDA network polymers. The corresponding profile of SQ109 also showed the similar endothermic peak. The peak should be derived from degradation of SQ109, as observed in perfect cage type silsesquioxane [16]. A solid residue about $40 \mathrm{wt} \%$ was observed after the heating to $480^{\circ} \mathrm{C}$ under the argon atmosphere. The perfect cage type silsesquioxane shows almost complete weight loss at around $420^{\circ} \mathrm{C}$ under nitrogen atmosphere [16]. The solid residue should be derived from hydrocarbon moieties of BDA in SQ109-BDA polymer network. 
Mechanical properties of SQ109-BDA network polymers were investigated by compression test. Stress-strain curves of the SQ109-BDA network polymers are shown in Figure 6. Young's modulus of the network polymer increased with increasing in monomer concentration of the reaction system, $4.3 \mathrm{kPa}$ (run 2, 15 wt\%), $14.0 \mathrm{kPa}$ (run 3, $25 \mathrm{wt} \%$ ), $81.2 \mathrm{kPa}$ (run 5, $45 \mathrm{wt} \%$ ), due to the increase of the space occupation by the polymer networks. SQ109-BDA network polymers with porous morphology, which were obtained from the reaction systems with lower monomer concentrations (runs 2 and 3 as shown in Figure 2(B) and Figure 2(C)), were unbreakable under the compression of $50 \mathrm{~N}$. By contrast, a non-porous polymer obtained from the reaction system with $45 \mathrm{wt} \%$ monomers (run 5 as shown in Figure 2(D) was broken at about $11 \%$ of strain. The porous structure should absorb the pressure, and induced soft and flexible features in the network polymers.

The SQ109-BDA porous polymer prepared by Irg184 (run 6) showed higher Young's modulus, $33.3 \mathrm{kPa}$, than the corresponding porous polymer prepared by AIBN (run 2). The globule size in the former porous polymer $(1.4 \mu \mathrm{m})$ was smaller than that in the latter porous polymer $(1.7-5.0 \mu \mathrm{m})$, as summarized in Table 1. The morphology of connected globules with small size should induce hard feature of the porous polymer, as previously reported in other porous polymers [5].

\subsection{SQ109-Diisocyanate Network Polymer}

Thiol-isocyanate reaction of SQ109 and HDI or MDI was conducted in some solvents at room temperature using TEA as a catalyst, as illustrated in Scheme 3 (B). For example, the reaction in DMF yielded homogeneous clear gel independent of monomer concentration in the reaction system, Table 2 runs $9-13$,

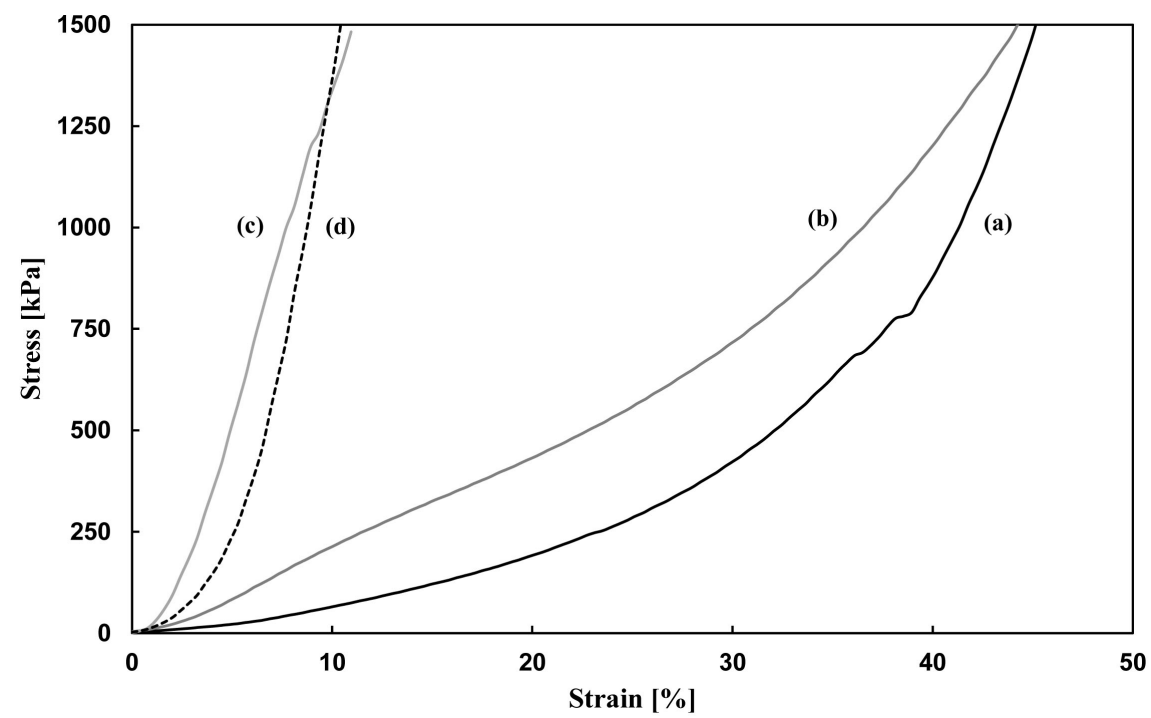

Figure 6. Stress-strain curves of SQ109-BDA network polymers, monomer concentration in the reaction system; (a) $15 \mathrm{wt} \%$ (run 2), (b) $25 \mathrm{wt} \%$ (run 3), or (c) $45 \mathrm{wt} \%$ (run 5) initiated by AIBN, and (d) $15 \mathrm{wt} \%$ initiated by Irg184 (run 6). 
Table 2. Synthesis of network polymers by thiol-isocyanate reaction of SQ109 with HDI, MDI.

\begin{tabular}{|c|c|c|c|c|c|c|}
\hline Run & Linker & $\begin{array}{l}\text { Monomer } \\
\text { conc. (wt\%) }\end{array}$ & $\begin{array}{l}\text { Solvent DMF/toluene } \\
\text { (vol.\%/vol.\%) }\end{array}$ & State & $\begin{array}{c}\text { SEM } \\
\text { observation }\end{array}$ & $\begin{array}{l}\text { Size }^{\mathrm{a}} \\
(\mu \mathrm{m})\end{array}$ \\
\hline 9 & HDI & 5 & $100 / 0$ & Clear gel & Bulk & \\
\hline 10 & HDI & 15 & $100 / 0$ & Clear gel & Bulk & \\
\hline 11 & HDI & 25 & $100 / 0$ & Clear gel & Bulk & \\
\hline 12 & HDI & 35 & $100 / 0$ & Clear gel & Bulk & \\
\hline 13 & HDI & 45 & $100 / 0$ & Clear gel & Bulk & \\
\hline 14 & HDI & 5 & $50 / 50$ & White gel & Porous & 1.3 \\
\hline 15 & HDI & 15 & $50 / 50$ & White gel & Porous & $<0.5$ \\
\hline 16 & HDI & 25 & $50 / 50$ & White gel & Porous & $<0.5$ \\
\hline 17 & HDI & 35 & $50 / 50$ & Clear gel & Bulk & \\
\hline 18 & HDI & 45 & $50 / 50$ & Clear gel & Bulk & \\
\hline 19 & MDI & 5 & $100 / 0$ & Clear gel & Bulk & \\
\hline 20 & MDI & 15 & $100 / 0$ & Clear gel & Bulk & \\
\hline 21 & MDI & 25 & $100 / 0$ & Clear gel & Bulk & \\
\hline 22 & MDI & 35 & $100 / 0$ & Clear gel & Bulk & \\
\hline 23 & MDI & 45 & $100 / 0$ & Clear gel & Bulk & \\
\hline 24 & MDI & 5 & $50 / 50$ & White gel & Porous & $<0.5$ \\
\hline 25 & MDI & 15 & $50 / 50$ & White gel & Porous & $<0.5$ \\
\hline 26 & MDI & 25 & $50 / 50$ & White gel & Porous & $<0.5$ \\
\hline 27 & MDI & 35 & $50 / 50$ & White gel & Porous & $<0.5$ \\
\hline 28 & MDI & 45 & $50 / 50$ & Clear gel & Bulk & \\
\hline
\end{tabular}

a averaged diameter of globule.

19 - 23. By contrast, some reactions in a mixed solvent of DMF and toluene, DMF/toluene $=50 / 50 \mathrm{vol} . \% / \mathrm{vol} . \%$, yielded white gel, as summarized in Table 2 runs $14-16,24-27$. Calculated solubility parameters of connection moiety in SQ109-HDI and SQ109-MDI network polymers are shown in Scheme 5. The calculated solubility parameter value of the boxed part in Scheme 5 formed by the addition of thiol with HDI or MDI is 14.1 or 15.9 (or 14.7) $\left(\mathrm{cal} / \mathrm{cm}^{3}\right)^{1 / 2}$, respectively. The SP of DMF or DMF/toluene (50/50 vol.\%/vol.\%) is 12.0 or 10.4 $\left(\mathrm{cal} / \mathrm{cm}^{3}\right)^{1 / 2}$, respectively. Relatively small difference in SP value between thio-urethane unit and DMF should form homogeneous phase, clear gel, in the polymerizations of SQ109 with HDI and MDI. Mixture solvent with toluene decreased the SP value, and the difference in SP values between the thio-urethane unit and the solvent became large, which should induce phase separation in the reaction system. The reaction system of SQ109-MDI with $35 \mathrm{wt} \%$ monomer concentration yielded white gel induced by phase separation. By contrast, the corresponding SQ109-HDI system formed clear gel. Lager difference in SP value 

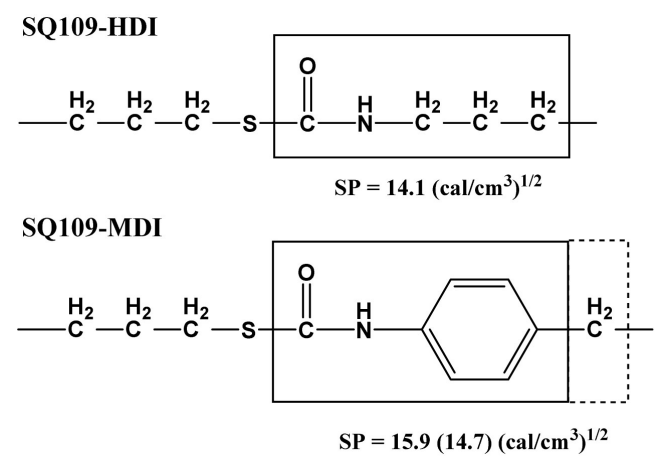

Scheme 5. Structure and SP value of connection moiety in SQ109-HDI and SQ109-MDI network polymers.

between thio-urethane unit of SQ109-MDI and the solvent in comparison with that of SQ109-HDI would induce the phase separation in the reaction with relatively high monomer concentration.

Figure 7 shows SEM images of the dried SQ109-HDI network polymers. The network polymer obtained from the reaction system containing $5 \mathrm{wt} \%$ monomers showed a porous structure, which was formed by connected sphere globules more than $1 \mu \mathrm{m}$ radius and co-continuous vacant space. The reaction systems containing 15 and $25 \mathrm{wt} \%$ monomers yielded porous polymers, which were formed by connected small particles less than $0.5 \mu \mathrm{m}$ radius. The morphology should be formed by polymerization induced phase separation via spinodal decomposition, as observed in SQ109-BDA network polymer. Averaged globule (particle) size of the porous polymer obtained from the reaction system with 5 wt\% monomers was $1.3 \mu \mathrm{m}$ and the size decreased, less than $0.5 \mu \mathrm{m}$, with increasing in the monomers concentration in the reaction systems, as observed in the porous polymers obtained from the reaction systems with $15 \mathrm{wt} \%$ and 25 $\mathrm{wt} \%$ monomers. The network polymers obtained from the reaction systems with 35 and $45 \mathrm{wt} \%$ monomers showed non-porous bulk morphology. The porous polymer obtained from the reaction system with $5 \mathrm{wt} \%$ monomers should be formed at the phase separation state of Figure 3(iv). The reaction systems with 15 or $25 \mathrm{wt} \%$ monomers should be fixed at earlier state of the phase separation, as shown in Figure 3(iii), due to the increase of the reaction rate, and the diameter of the particles became small. Further increase of the monomer concentration in the reaction systems ( 35 or $45 \mathrm{wt} \%$ ) should increase the reaction rate and formed the network polymers before phase separation as clear gels, as shown in Figure 3(i), and showed non-porous morphology.

SEM images of the dried SQ109-MDI network polymers are shown in Figure 8. All the network polymers obtained from the reaction systems containing 5,15 , 25 and 35 wt\% monomers showed a morphology, which was formed by connected small particles. Sizes of particles were less than $0.5 \mu \mathrm{m}$, even that of the porous polymer obtained from the reaction system with low monomer concentration (5 wt\%). That means SQ109-MDI network polymer tends to form smaller particles than SQ109-HDI network polymer. Molecular structure of SQ109-HDI 


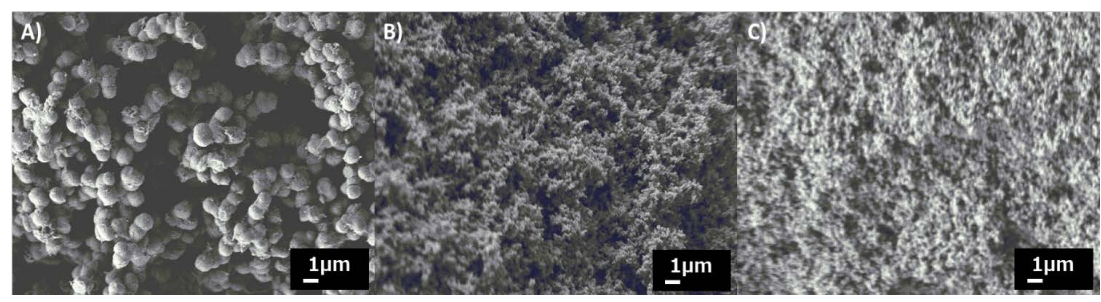

Figure 7. SEM images of SQ109-HDI polymers synthesized by thiol-isocyanate reaction, solvent: $\mathrm{DMF} /$ toluene $=50 / 50$ vol.\%/vol.\%, monomer concentration: (A) $5 \mathrm{wt} \%$; (B) 15 wt\%; and (C) $25 \mathrm{wt} \%$.

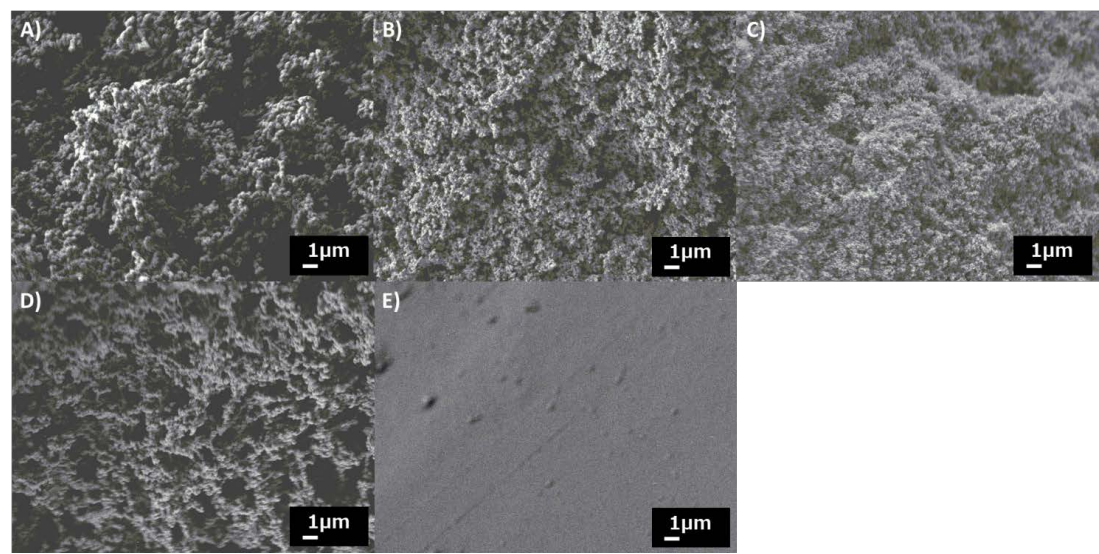

Figure 8. SEM images of SQ109-MDI polymers synthesized by thiol-isocyanate reaction, solvent: DMF/toluene $=50 / 50$ vol.\%/vol.\%, monomer concentration: (A) $5 \mathrm{wt} \%$; (B) 15 wt\%; (C) $25 \mathrm{wt} \%$; (D) $35 \mathrm{wt} \%$ and (E) $45 \mathrm{wt} \%$.

and SQ109-MDI polymers was studied by FT-IR spectroscopy, as shown in Figure 9. The absorption peaks at around $2500-2600 \mathrm{~cm}^{-1}$ derived from NCO (in HDI or MDI) and $2560 \mathrm{~cm}^{-1}$ derived from thiol group (in SQ109) were almost disappeared in the spectra of the resulting network polymers. Absorption peaks derived from Si-O-Si unit of SQ109 and thio-urethane bond were detected at around $1050-1200 \mathrm{~cm}^{-1}$ and $3300 \mathrm{~cm}^{-1}$, respectively. SQ109-MDI polymer showed an absorption peak derived from phenyl group at around $1400 \mathrm{~cm}^{-1}$. These results showed the thiol-isocyanate reactions successfully occurred in both the reaction systems. One explanation of formation of the small particle size in SQ109-MDI network polymer is that high reactivity of NCO in MDI, as generally known as a monomer of polyurethane. High reaction rate of MDI should fix the porous structure at more early state of Figure 3(iii), which decreased the size of the particles. Another possibility is larger difference in SP values between connection moiety in SQ109-MDI network polymer, $15.9\left(\mathrm{cal} / \mathrm{cm}^{3}\right)^{1 / 2}$, and DMF/ toluene solvent, $10.4\left(\mathrm{cal} / \mathrm{cm}^{3}\right)^{1 / 2}$, in comparison with that in SQ109-HDI network polymer, $14.1\left(\mathrm{cal} / \mathrm{cm}^{3}\right)^{1 / 2}$, as shown in Scheme 5. Lower affinity between SQ109-MDI network and the solvent should fix the phase separation state at earlier stage. The SQ109-MDI reaction system with $45 \mathrm{wt} \%$ monomers formed non-porous morphology polymer, which should form the polymer network before phase separation, as shown in Figure 3(i). 


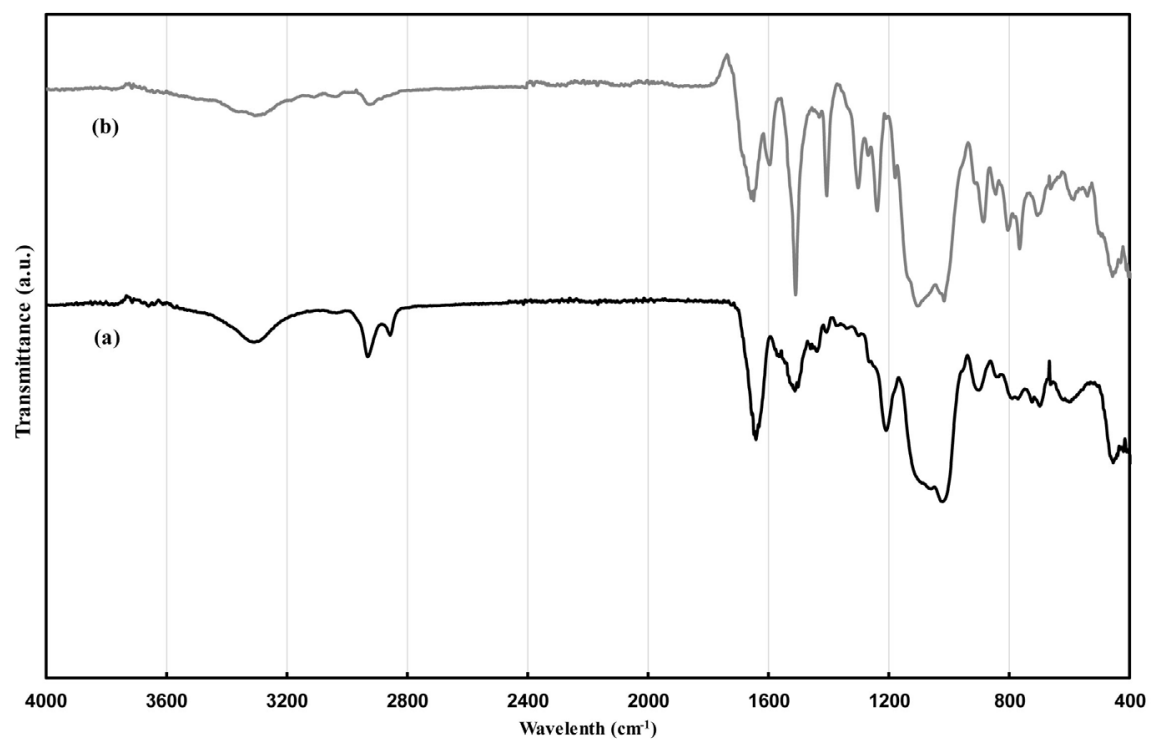

Figure 9. FT-IR spectra of (a) SQ109-HDI and (b) SQ109-MDI network polymers obtained from the reaction systems in DMF/toluene (50/50 vol.\%/vol.\%), monomer concentration in the reaction solution; $25 \mathrm{wt} \%$.

TGA and DSC profiles of SQ109-HDI and SQ109-MDI porous polymers are shown in Figure 10. Weight loss of SQ109-HDI (run 15) and SQ109-MDI (run 16) network polymers began at round $260^{\circ} \mathrm{C}$ ( $5 \mathrm{wt} \%$ weight loss was attained at $266^{\circ} \mathrm{C}$ and $268^{\circ} \mathrm{C}$, respectively) in TGA. Multiple endothermic peaks were detected in the range from $250^{\circ} \mathrm{C}$ to $400^{\circ} \mathrm{C}$ in DTA and DSC profiles. These phenomena should be derived from thermal degradation of thio-urethane bond formed by the addition reaction of thiol and isocyanate [17]. Heat flows of the endothermic peaks at round $350^{\circ} \mathrm{C}-360^{\circ} \mathrm{C}$ of the SQ109-HDI porous polymers (Figure 10(B) (b) and (c)) were larger than those of the SQ109-MDI porous polymers (Figure 10(B) (d) and (e)) in the DSC profiles. One explanation on the results is that molecular structure in diisocyanate, methylene (HDI) or phenyl (MDI) group, would affect the thermal degradation behavior of the adjoining thio-urethane. Broad shoulder endothermic peaks, which were derived from degradation of SQ109, were detected at around $420^{\circ} \mathrm{C}$ in the DSC profiles, as observed in SQ109-BDA porous polymers. Solid residues about $50 \mathrm{wt} \%$ after the heating to $480^{\circ} \mathrm{C}$ should be derived from hydrocarbon moieties of HDI or MDI in the polymer networks with SQ109, as observed in SQ109-BDA polymer network.

Mechanical properties of SQ109-HDI and SQ109-MDI porous polymers were investigated by compression test. All the porous polymers were very hard independent of the monomer concentration in the reaction systems, and showed high Young's modulus more than $50 \mathrm{kPa}$ and were unbreakable under the compression of $50 \mathrm{~N}$. Reproducibility and qualitative discussion in the Young's modulus of the SQ109-HDI and SQ109-MDI porous polymers were difficult due to very hard property. Both chemical structure, interaction of thio-urethane groups in the polymer network, and morphology, small globules or particles less than $0.5 \mu \mathrm{m}$, of these porous polymers should induce too high Young's modulus 


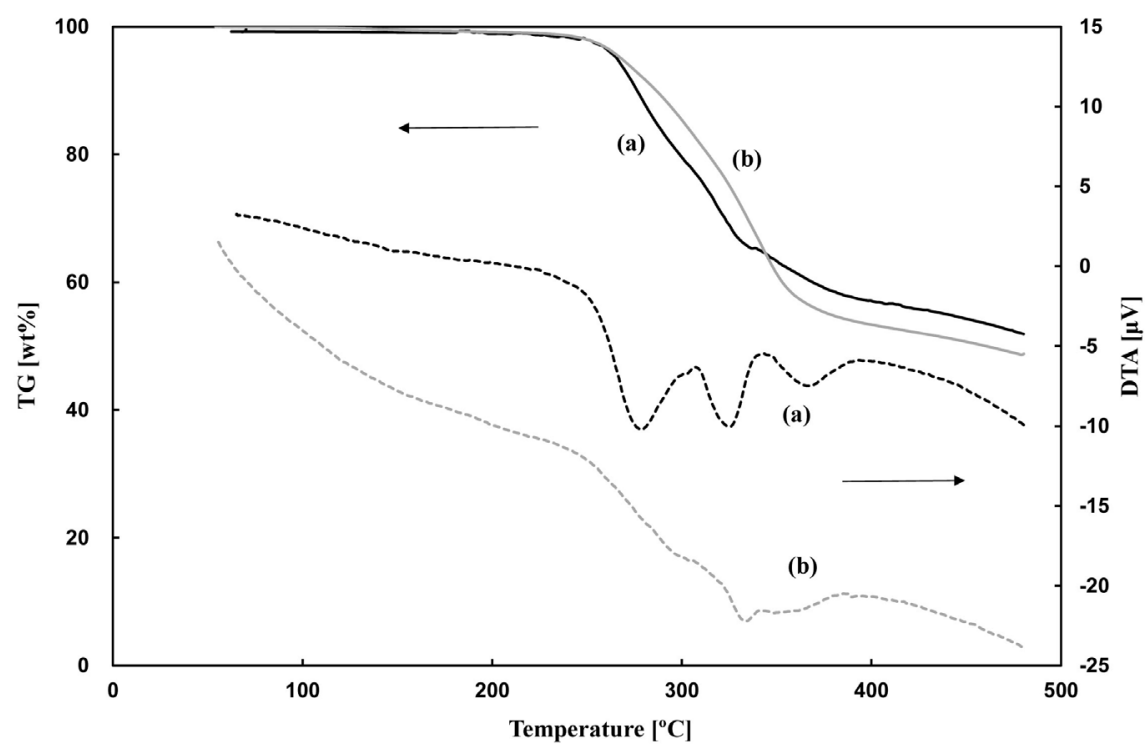

(A)

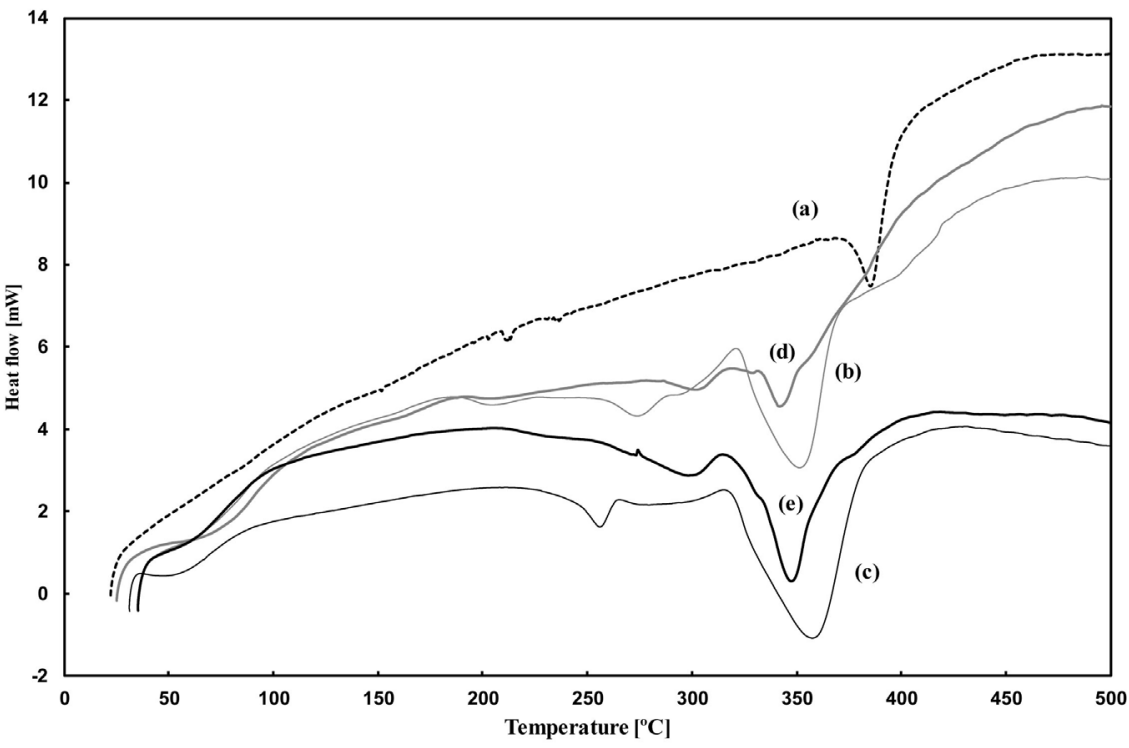

(B)

Figure 10. (A) TG-GTA profile of (a) SQ109-HDI (run 15) and (b) SQ109-MDI (run26); (B) DSC profiles of SQ109 (a) for a reference and SQ109-HDI network polymers; monomer concentration in the reaction system, (b) $15 \mathrm{wt} \%$ (run 15), (c) $25 \mathrm{wt} \%$ (run 16), and SQ109-MDI network polymers; monomer concentration in the reaction system, (d) 15 wt\% (run 25), (e) 25 wt\% (run 26).

to evaluate with the compression test.

\section{Conclusions}

Organic-inorganic hybrid network polymers were synthesized by thiol-ene reaction or thiol-isocyanate reaction of a thiol-functionalized random type silsesquioxane, SQ109, and diacrylate, diolefin, or diisocynate. The thiol-ene reaction of SQ109 and BDA in toluene initiated by AIBN successfully yielded the porous 
polymers. Monomer concentration in the reaction system strongly affected morphology and size of the porous structure. The porous polymers, which were obtained from the reaction systems with $5-25 \mathrm{wt} \%$ of the monomers, were composed by connected globules less than $5 \mu \mathrm{m}$ of diameters. The reaction system with $35 \mathrm{wt} \%$ monomers yielded the porous polymer composed by co-continuous structure (hole) and globules, which should be formed at early stage of the reaction. A photo-initiated reaction using Irg184 also yielded the porous polymer. The reactions with HDA or HD formed gels, which showed non-porous bulky structure. Effective permeation of toluene into SQ109-HDA network, or high affinity between connecting moiety of SQ109-HD and toluene would promote homogeneous phase. Weight loss of SQ109-BDA porous polymer began at round $300^{\circ} \mathrm{C}$ derived from thermal degradation of ester group of BDA moiety in the polymer network. SQ109-BDA porous polymer with the morphology composed by small globules showed high Young's modulus.

Thiol-isocyanate reaction of SQ109 and HDI or MDI in DMF formed clear gels independent of the monomer concentration. The corresponding reactions in DMF/toluene mixed solvent with low monomer concentrations yielded the porous polymers. Difference in SP values between the polymer network and solvent was important to control of the phase in the reactions. The porous polymers showed the morphology formed by connected globules, and their diameters tended to become small with increasing in monomer concentration of the reaction system. SQ109-MDI network polymer showed smaller globules, which should be formed at earlier stage of spinodal decomposition than that of SQ109HDI network polymer due to the higher reaction rate with MDI. Thermal degradation of SQ109-HDI and SQ109-MDI porous polymers occurred at round $260^{\circ} \mathrm{C}$ and showed endothermic peak at around $350^{\circ} \mathrm{C}$ derived from degradation of thio-urethane bond. Both the SQ109-HDI and SQ109-MDI porous polymers showed enough high mechanical properties derived interaction of thio-urethane bonds in the polymer network and small globule size of the porous morphology.

As mentioned above, click type addition reactions of a commercially available random type silsesquioxane, SQ109, as a joint monomer, and conventional $\alpha$, $\omega$-alkyl diacrylate or diisocyanate, as linker monomer, are usable methods to synthesize organic-inorganic hybrid porous polymers. Phase and morphology of the network polymers can be controlled by the reaction conditions, especially nature of solvent, structure of linker monomer, and monomer concentration. Additional porogen was not necessary in the present methods to prepare the hybrid porous polymers, and the resulting porous polymers were easily purified by replacement the solvent. Most of the porous polymers were formed by connected globules or small particles, whose size could be controlled by monomer concentration in the reaction system. As the next step, we are studying application of the organic-inorganic hybrid porous polymers. The globule or particle size of the resulting porous polymers, less than $1 \mu \mathrm{m}$, is closed to wavelength of visible light. We are also trying to synthesize the porous polymer with narrow size distribution in the morphology, which would be useful for photonic crystal, 
and the results will be reported elsewhere.

\section{Conflicts of Interest}

The authors declare no conflicts of interest regarding the publication of this paper.

\section{References}

[1] Naga, N., Oda, E., Toyota, A., Horie, K. and Furukawa, H. (2006) Tailored Synthesis and Fundamental Characterization of Organic-Inorganic Hybrid Gels by Means of a Hydrosilylation Reaction. Macromolecular Chemistry and Physics, 207, 627-635. https://doi.org/10.1002/macp.200500501

[2] Naga, N., Oda, E., Toyota, A. and Furukawa, H. (2007) Mesh Size Control of Organic-Inorganic Hybrid Gels by Means of a Hydrosilylation Co-Gelation of Siloxane or Silsesquioxane and $\alpha, \omega$-Non-Conjugated Dienes. Macromolecular Chemistry and Physics, 208, 2331-2338. https://doi.org/10.1002/macp.200700184

[3] Naga, N., Kihara, Y., Miyanaga, T. and Furukawa, H. (2009) Synthesis of Organic-Inorganic Hybrid Gels from Siloxane or Silsesquioxane and $\alpha, \omega$-Nonconjugated Dienes by Means of a Photo Hydrosilylation Reaction. Macromolecules, 42, 3454 3462. https://doi.org/10.1021/ma802745x

[4] Naga, N., Nagino, H. and Furukawa, H. (2016) Synthesis of Organic-Inorganic Hybrid Gels by Means of Thiol-Ene and Azide-Alkene Reactions. Journal of Polymer Science Part A: Polymer Chemistry, 54, 2229-2238.

https://doi.org/10.1002/pola.28096

[5] Naga, N., Fujioka, S., Inose, D., Ahmed, K., Nageh, H. and Nakano, T. (2020) Synthesis and Properties of Porous Polymers Synthesized by Michael Addition Reactions of Multi-Functional Acrylate, Diamine, and Dithiol Compounds. RSC Advances, 20, 60-69. https://doi.org/10.1039/C9RA09684A

[6] Naga, N., Michida, R., Kudo, S., Nagami, Y., Moriyama, K., Nageh, H., Furukawa, H. and Nakano, T. (2019) Synthesis of Joint-Linker Type Gels and Porous Polymers by Addition Reactions of Multi-Functional Thiol and Alkyl Diacrylate, Diisocyanate Compounds. Materials Today Communications, 18, 153-162.

https://doi.org/10.1016/j.mtcomm.2018.11.013

[7] Liu, Z., Ou, J. and Zou, H. (2016) Click Polymerization for Preparation of Monolithic Columns for Liquid Chromatography. Trends in Analytical Chemistry, 82, 89-99. https://doi.org/10.1016/j.trac.2016.05.016

[8] Murphy, A.R., Ghobrial, I., Jamshidi, P., Laslett, A., O’brien, C.M. and Cameron, N.R. (2017) Tailored Emulsion-Templated Porous Polymer Scaffolds for iPSC-Derived Human Neural Precusor Cell Culture. Polymer Chemistry, 8, 6617-6627. https://doi.org/10.1039/C7PY01375B

[9] Alves, F. and Nischang, I. (2013) Tailor-Made Hybrid Organic-Inorganic Porous Materials Based on Polyhedral Oligomeric Silsesquioxane (POSS) by the StepGrowth Mechanism of Thiol-Ene "Click" Chemistry. Chemistry-A European Journal, 19, 17310-17313. https://doi.org/10.1002/chem.201303759

[10] Imoto, H., Ishida, A., Hashimoto, M., Mizoue, Y., Yusa, S. and Naka, K. (2019) Soluble Network Polymers Based on Trifunctional Open-Cage Silsesquioxanes. Chemistry Letters, 48, 1266-1269. https://doi.org/10.1246/cl.190536

[11] Li, C., Tan, J., Li, H., Yin, D., Gu, J., Zhang, B. and Zhang, Q. (2015) Thiol-Isocyanate Click Reaction in a Pickering Emulsion: A Rapid and Efficient Route to En- 
capsulation of Healing Agents. Polymer Chemistry, 6, 7100-7111.

http://pubs.rsc.org/-/content/articlehtml/2015/py/c5py01323b

https://doi.org/10.1039/C5PY01323B

[12] Dailing, E.A., Nair, P., Setterberg, W.K., Kyburz, K.A., Yang, C., D’Ovidio, T., Anseth, K.S. and Stansbury, J.W. (2016) Combined, Independent Small Molecule Release and Shape Memory via Nanogel-Coated Thiourethane Polymer Networks. Polymer Chemistry, 7, 816-825. https://doi.org/10.1039/C5PY01464F http://pubs.rsc.org/en/Content/ArticleLanding/2016/PY/c5py01464f\#!divAbstract

[13] Tan, J., Li, C., Li, H., Zhang, H., Gu, J., Zhang, B., Zhang, H. and Zhang, Q. (2015) Water-Borne Thiol-Isocyanate Click Chemistry in Microfluidics: Rapid and Energy-Efficient Preparation of Uniform Particles. Polymer Chemistry, 6, 4366-4373. http://pubs.rsc.org/en/Content/ArticleLanding/2015/PY/C5PY00412H\#!divAbstract https://doi.org/10.1039/C5PY00412H

[14] Jia, Y., Shi, B., Jin, J. and Li, J. (2019) High Refractive Index Polythiourethane Networks with High Mechanical Property via Thiol-Isocyanate Click Reaction. Polymer, 180, Article ID: 121746. https://doi.org/10.1016/j.polymer.2019.121746

[15] Fedors, R.F. (1974) A Method for Estimating Both the Solubility Parameters and Molar Volumes of Liquids. Polymer Engineering \& Science, 14, 147-154.

https://doi.org/10.1002/pen.760140211

[16] Blanco, I., Abate, L., Bottino, F.A., Bottino, P. and Chiacchio, M.A. (2012) Thermal Degradation of Differently Substituted Cyclopentyl Polyhedral Oligomeric Silsesquioxane (CP-POSS) Nanoparticles. Journal of Thermal Analysis and Calorimetry, 107, 1083-1091. https://doi.org/10.1007/s10973-011-1848-3

[17] Rogulska, M., Kultys, A. and Olszewska, E. (2013) New Thermoplastic Poly(thiourethane-urethane) Elastomers Based on Hexane-1,6-diyl Diisocyanate (HDI). Journal of Thermal Analysis and Calorimetry, 114, 903-916.

https://doi.org/10.1007/s10973-013-3007-5 